\title{
Robustness assessment of China's high-speed railway transportation network
}

\author{
Zhuo Wang ${ }^{\text {a, b }}$, Haowen Yan ${ }^{\text {a, b, }}$,Yuhan Huang ${ }^{\text {a, b }}$,Yazhen Li $^{\text {c, d }}$ \\ a Faculty of Geomatics, Lanzhou Jiaotong University, Lanzhou 730070, China, $1078095001 @$ qq.com, H. \\ Yan,haowen2010@gmail.com,Y.Huang,435845463@qq.com \\ b Gansu Provincial Engineering Laboratory for National Geographic State Monitoring, Lanzhou 730070, China \\ 1078095001@qq.com, H.Yan,haowen2010@gmail.com,Y.Huang,435845463@qq.com \\ c Northwest Institute of Eco-Environment and Resources, CAS, Lanzhou 730000,China, Y. Li, liyazhen@lzb.ac.cn \\ d University of Chinese Academy of Sciences, Beijing 100049, China, Y. Li, liyazhen@lzb.ac.cn \\ * Corresponding author: H. Yan, haowen2010@gmail.com
}

Keywords: High speed rail transport; complex networks; Robustness

\begin{abstract}
:
The robustness of the high-speed rail transportation network is closely related to the passenger transportation efficiency, and is also a concrete manifestation of the anti-jamming capability of the high-speed railway transportation system. By the end of 2017 , the business mileage of Chinese high-speed railway has reached $25,164 \mathrm{~km}$, the number of passengers has reached 175.216 million, accounting for $56.8 \%$ of railway passenger traffic; the number of passenger turnover has reached 587.56 billion peroson-km, accounting for $43.7 \%$ of railway passenger traffic turnover.As of 2018 On December 4th, the country operate 4,251 high-speed railway trains every day. At the same time, passenger transportation efficiency of high-speed railways is highly susceptible to sudden events such as natural disasters. Therefore, quantitative assessment of anti-jamming capability of high-speed railway network, and the propose of specific improvements, are of great practical significance to improve passenger transport efficiency, enhance scientific decision-making high-speed rail transport infrastructure investments.
\end{abstract}

This paper used the the high-speed train running data on December 4, 2018 to analyze the work.Its high-speed railway network schematic is shown in Figure 1.

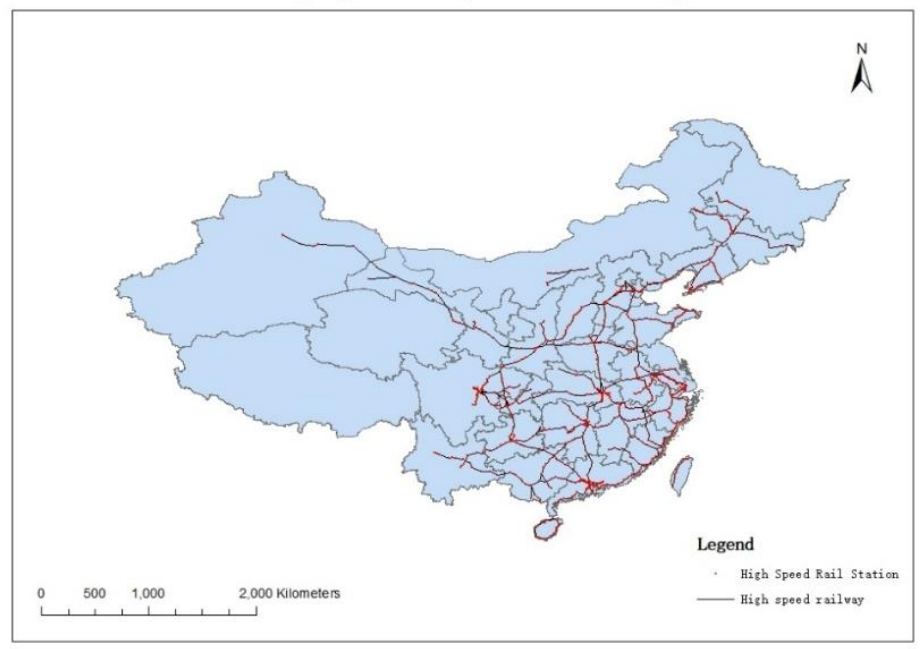

Fig.1 Chinese high speed railway road network

The Scale-free property of networks means that the node degree of networks obeys power-law distribution. It is generally believed that scale-free networks exhibit robustness against random faults and vulnerability to deliberate attacks. In this paper, the regularity of node degree change is expressed by distribution function $\mathrm{P}(\mathrm{k})$. As shown in the degree distribution of double logarithmic coordinate system as shown in Figure 2, the network has a great goodness of fit and better obeys power law score.That is to say, the network is a scale-free network.

In this paper, random attacks and deliberate attack simulations are used to observe the condition of the structure of China's high-speed railway network, and in order to measure the robustness of high-speed rail network. Among them, the formula refers to random $r \cdot$ andom delete a node from the network; deliberate attack is to delete nodes according to their importance. Attack strategies for node degree and intermediate centrality are used to determine which nodes are selected for each simulated attack (the lower the importance, the smaller the impact on the network). According to the three attack strategies for the high-speed railway transportation network, experiments are carried out to carry out 
continuous simulation attacks on the network: in each time, a node in the network and all its connected edges are deleted, the relative average shortest path and average clustering coefficient of the network at this time are counted. And the degree of fragmentation, looping until all nodes in the network are deleted, that is to say, the network is completely invalid. In Fig. 3, Fig. 4 and Fig. 5, the $\mathrm{X}$ axis represents the number of attack nodes, and the $\mathrm{Y}$ axis represents the current network structure feature metric.

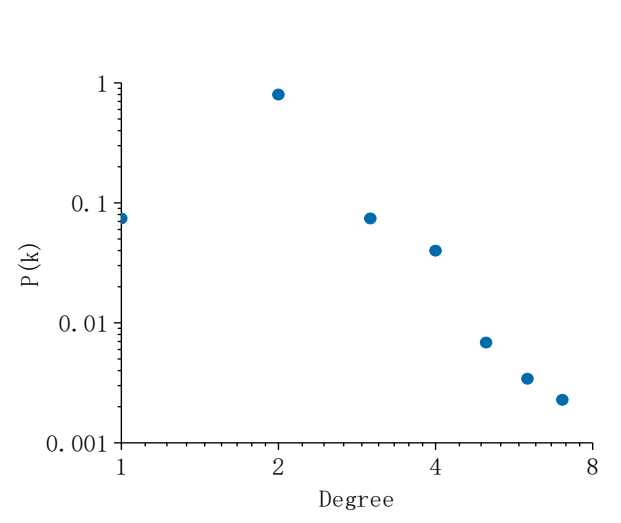

Fig.2 The degree distribution

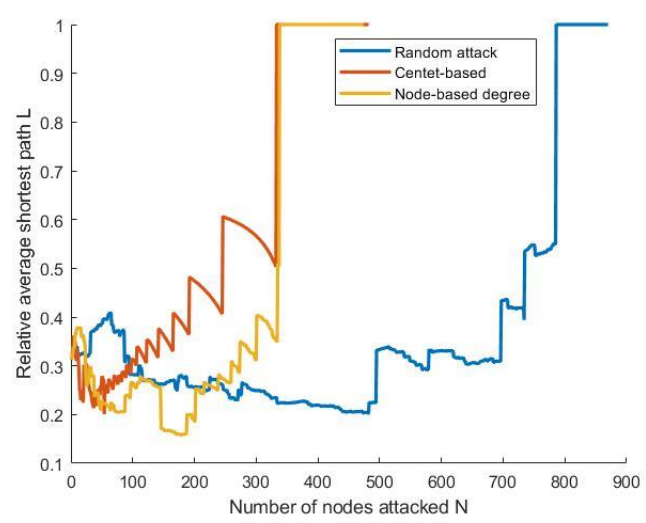

Fig.4 Relative average shortest distance change

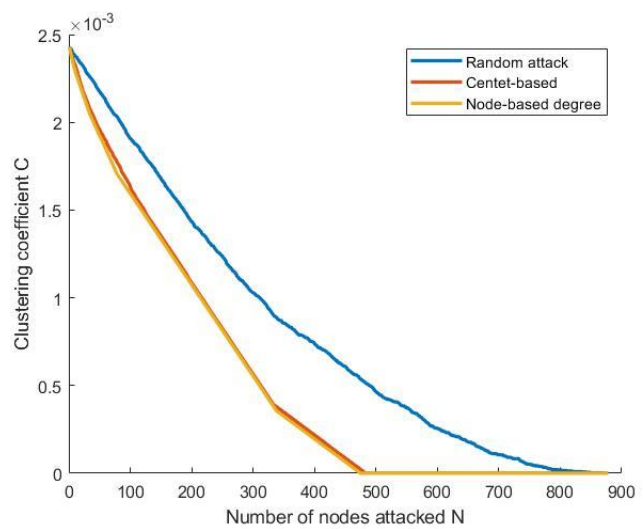

Fig.3 Clustering coefficient change

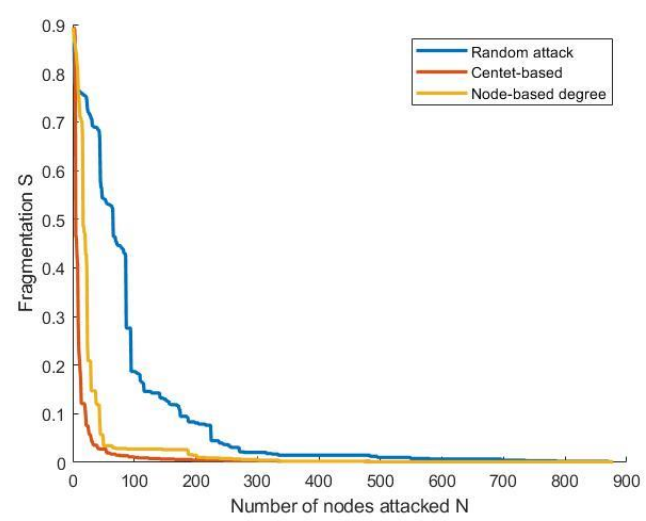

Fig.5 Change in fragmentation

It can be seen that the intentional attack based on the centrality of node mediation has the most serious damage to the network, that is, in other word, the state of splitting and crashing of the network.. For deliberate attacks, China's high-speed railway transport network is very fragile, and attacking nodes with high intermediary centrality value is the most destructive to China's high-speed railway transport network. Therefore, in order to ensure the normal operation of passenger transport we need to focus on ensuring the stability of the sites with the highest intermediary value. This paper ranks the sites according to the value of intermediary centrality. Figure 6 shows the top 10 sites in China's high-speed rail transport network with centrality as intermediaries.

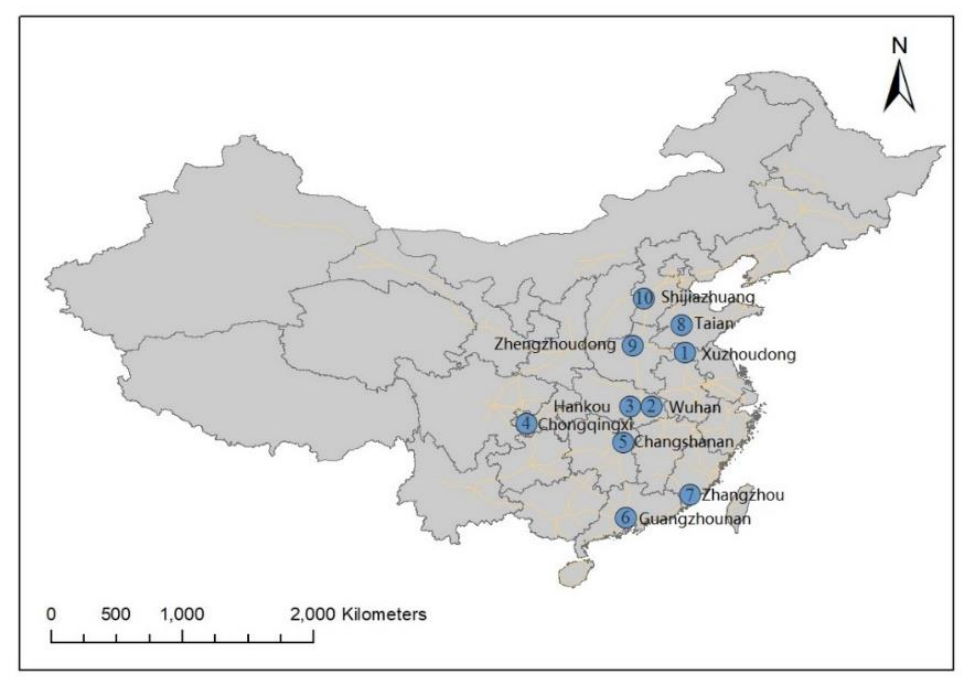

Fig.6 Top 10 of stations based on betweenness centrali 